\title{
Polymeric micelles to deliver photosensitizers for photodynamic therapy
}

\author{
Cornelus F. van Nostrum* \\ Department of Pharmaceutics, Utrecht Institute for Pharmaceutical Sciences, Sorbonnelaan 16, P.O. Box 80082, \\ 3508 TB Utrecht, The Netherlands
}

Received 6 June 2003; accepted 30 September 2003

\begin{abstract}
Polymeric micelles are emerging as attractive drug delivery systems. Hydrophobic drugs including photosensitizers for photodynamic therapy can be covalently bound or physically entrapped in the core of the micelles and thus be systemically delivered to, for example, tumors using passive or active targeting strategies. Polymers used for photosensitizer encapsulation include pluronics, poly(ethylene glycol) (PEG)-lipid conjugates, and $\mathrm{pH}$-sensitive poly $(N$-isopropylacrylamide) based micelles or polyion complex (PIC) micelles. This paper reviews the results obtained so far, including drug loading, biodistribution studies, and therapeutic efficiency. The $\mathrm{pH}$-sensitive micelles appear to be promising candidates for photosensitizer delivery.

(c) 2003 Elsevier B.V. All rights reserved.
\end{abstract}

Keywords: Polymeric micelles; Photodynamic therapy; pH-responsive; Pluronics; Polyion complex micelles; Poly( $N$-isopropylacrylamide); Biodistribution

\section{Contents}

1. Introduction . . . . . . . . . . . . . . . . . . . . . . . . . . . 10

2. Pluronics and PEG-lipid formulations . . . . . . . . . . . . . . . . . . . . . . . . . . . 10

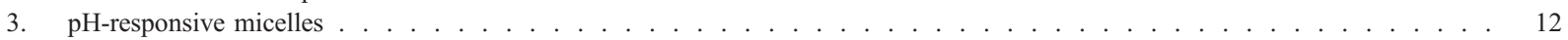

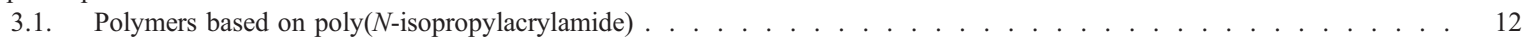

3.1.1. Micelle formation and drug loading . . . . . . . . . . . . . . . . . . . . . . . . . . . . . 12

3.1.2. In vitro evaluation . . . . . . . . . . . . . . . . . . . . . . . . . . . . 13

3.1.3. In vivo evaluation . . . . . . . . . . . . . . . . . . . . . . . . . . . 13

3.2. Polyion complex micelles . . . . . . . . . . . . . . . . . . . . . . . . . . . . . . . 14

4. Summary and future prospects . . . . . . . . . . . . . . . . . . . . . . . . . . 14

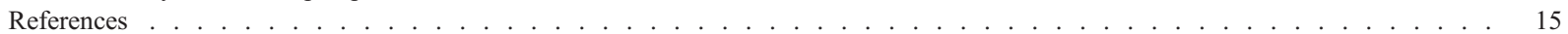

* Tel.: +31-30-253-6970; fax: +31-30-251-7839.

E-mail address: C.F.vanNostrum@pharm.uu.nl (C.F. van Nostrum). 


\section{Introduction}

Colloidal carriers are frequently used to transport and deliver drugs through the body for the reason of protecting the drug against degradation and/or excretion, to prevent adverse side effects of toxic drugs, or to accomplish targeted drug delivery. Examples of such carriers are micro/nanospheres, polymer-drug conjugates, liposomes, and (polymeric) micelles. After polymeric micelles were first proposed as drug carriers by Ringsdorf in 1984 [1], they have been emerging as a convenient carrier system. Some recently published papers provide excellent reviews on the use of polymeric micelles as drug carriers in general [2-5]. In the present contribution, I will focus on the application to deliver photosensitizers.

Polymeric micelles are formed in aqueous solution from amphiphilic block or graft copolymers. They contain hydrophobic segments, which form the core of the micelles, while the soluble segments form the corona, as shown schematically in Fig. 1. Polymeric micelles have been used to carry hydrophobic drugs, which are physically entrapped in and/or covalently bound to the hydrophobic core. Usually, physical entrapment is achieved by electrostatic interaction between drug and polymer (the resulting particles are called polyion complex (PIC) micelles [6]), by dialysis from an organic solvent, or by oil-in-water emulsion procedures. For drug delivery purposes, large variations in the composition of the core have been reported, e.g. polyesters [7-10], poly(amino acids) [11-13], poly(meth)acrylates [14], and poly(acrylamides) [15]. However, the corona has almost exclusively been constituted from poly(ethylene glycol) (PEG), because it is a highly biocompatible polymer which show little or no undesirable interactions with proteins and cells. PEG is frequently used to 'shield'

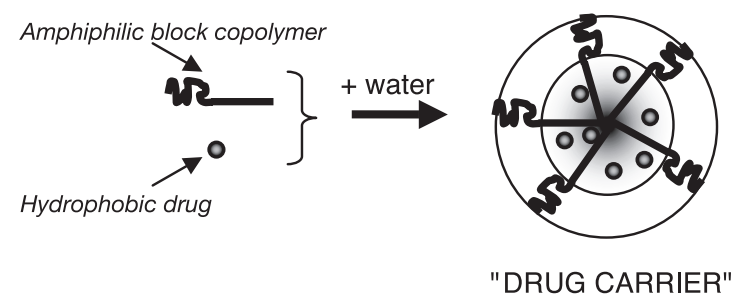

Fig. 1. Schematic representation of the micelle formation and drugloading of amphiphilic block copolymers in water. colloidal drug carriers from its environment in order to extend the residence time in the blood circulation [16].

There are a number of reasons why polymeric micelles are interesting as drug carriers. As a solubilizing agent for hydrophobic drugs they have a clear advantage over low molecular weight surfactants in view of the higher stability of the micelles. This higher stability is reflected in terms of the usually very low critical micelle concentration (CMC) of polymeric surfactants [4]. This means that polymeric micelles are resistant to dilution effects, upon for example i.v. administration of the drug formulation. Another important characteristic of micelles, when compared with, e.g. microspheres or many liposomal formulations, is their small and uniform particle size. In theory, particle sizes can go down to the order of $10 \mathrm{~nm}$ for non-loaded polymeric micelles. This size is still large enough to accomplish passive targeting to, e.g. tumors and inflamed tissues by the so-called enhanced permeation and retention (EPR) effect [17]. As said above, the hydrophilic corona of the micelles may prevent interaction with blood components. This characteristic and their small size will prevent recognition by proteins and macrophages, and thus long circulation times in the blood stream may be achieved [18]. Finally, active targeting is possible by modifying the peripheral chain ends of the polymers with targeting ligands $[19,20]$. For the release of the drugs once the micelles have reached their targets, degradable or stimuli-responsive micelles have been developed [15,21-23].

Since many photosensitizers usually display some toxicity against healthy cells and tissues, carriers are preferentially required to deliver them at the pathogenic sites by passive or active targeting [24]. Since many photosensitizers are insoluble in water, polymeric micelles are useful as a solubilization and delivery vehicle. This paper will review the work that has been done so far in this area.

\section{Pluronics and PEG-lipid formulations}

Pluronics (poloxamers) are commercially available water-soluble triblock copolymers of poly(ethylene oxide) and poly(propylene oxide) (therefore, often abbreviated as PEO-PPO-PEO), and have been frequently used as a solubilization agent in drug formulations [25]. Hioka et al. studied the use of pluronic 
P123 to solubilize a benzoporphyrin derivative (B-ring isomer, Fig. 2), aiming at photodynamic therapy [26]. Benzoporphyrin loading was done by hydration of a solid film obtained from organic solution containing the polymer and the photosensitizer. Above the CMC of $\mathrm{P} 123$ (i.e. $200 \mathrm{mg} \mathrm{l}^{-1}$ at $30{ }^{\circ} \mathrm{C}, 0.01 \mathrm{M}$ phosphate buffer $\mathrm{pH}$ 7.3) the photosensitizer is present in its

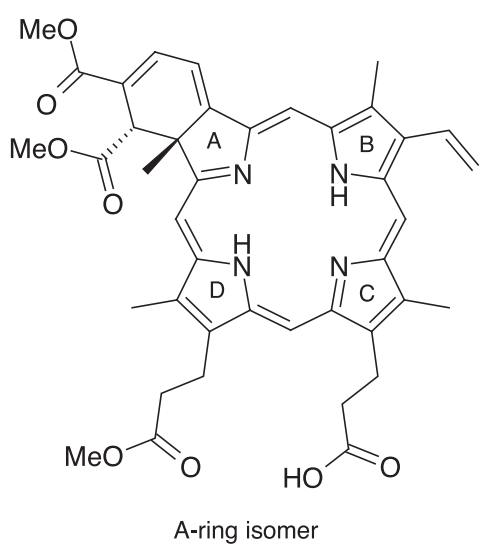

monomeric form in the core of the micelle at a benzoporphyrin concentration of $1 \mathrm{mg} \mathrm{ml}^{-1}$, while aggregates are formed in water below the CMC. This observation is important since aggregated photosensitizers have low quantum yields of light absorption and cause inefficient singlet oxygen production. The formation of stable micelles was reported at high $(10 \% \mathrm{w} /$

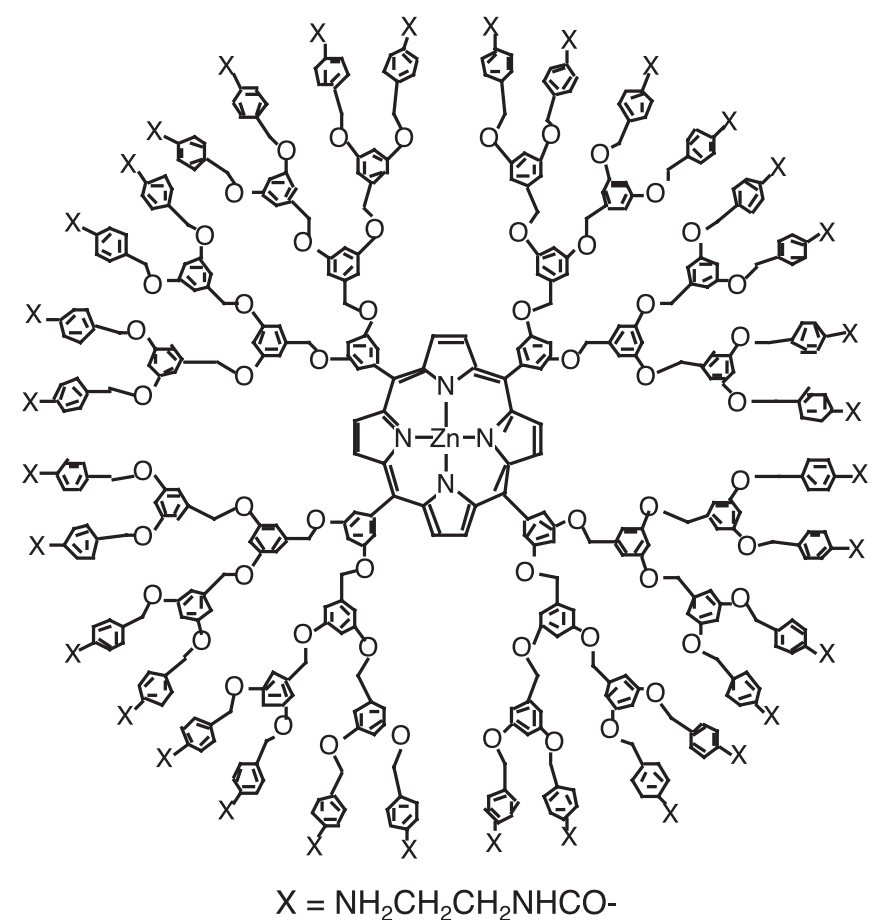

Fig. 2. Structural formulas of A- and B-ring isomers of benzoporphyrin (top) and zinc porphyrin dendrimer (bottom). 
v) P123 concentration with size ranging from 15 to 20 $\mathrm{nm}$. Low pluronic concentrations $(0.2 \%$ or less $)$ showed partly aggregated micelles. A minimum concentration of $4.8 \%$ was required to solubilize $1 \mathrm{mg}$ $\mathrm{ml}^{-1}$ of the benzoporphyrin, corresponding to a molar ratio $\mathrm{P} 123$ :benzoporphyrin $=6$ and a drug loading of approximately $2 \%(\mathrm{w} / \mathrm{w})$.

To date, no in vitro or in vivo studies have been reported yet towards the application of the pluronic-benzoporphyrin formulations in photodynamic therapy.

Micelle formulations of benzoporphyrin A- and Bring isomers were prepared in a similar way by Zhang et al. using methoxypoly(ethylene glycol) (molecular weight $2000 \mathrm{Da}$ ) covalently attached to the lipid distearoylphosphatidylethanolamine ( $\mathrm{mPEG}_{2000}$-DSPE) as the surfactant [27]. CMC was lower than P123 (i.e. approximately $50 \mathrm{mg}^{-1}$ at room temperature in 0.02 $\mathrm{M}$ phosphate buffer $\mathrm{pH} 8.5$ ), and increased slightly by addition of benzoporphyrin. $1 \mathrm{mg} \mathrm{ml}^{-1}$ of the B-ring benzoporphyrin was completely solubilized at a $\mathrm{mPEG}_{2000}$-DSPE:benzoporphyrin molar ratio of 5:1, the aggregation state of the photosensitizer depending on the $\mathrm{pH}$ of the solution; the monomer/dimer ratio increased from 0.8 in distilled water to 11.5 in phosphate buffer $\mathrm{pH} 8.5$, probably as a result of increasing degree of deprotonation of the benzoporphyrin above its $\mathrm{p} K_{\mathrm{a}}(\mathrm{pH}$ 6.5). Further increase of the monomer content was obtained by increasing the polymer/drug ratio.

In vivo tumor regression was studied with $\mathrm{mPEG}_{2000}$-DSPE:benzoporphyrin (6:1) formulations after i.v. injection of the A- or B-ring derivative (single dose of $1.4 \mu \mathrm{mol} \mathrm{kg}{ }^{-1}$ ) in DBA/2 mice carrying a rhabdosarcoma (M1) tumor, followed by exposure to $690 \mathrm{~nm}$ light after specific time points $(15-180 \mathrm{~min}$ post-injection). I.v. injection of the B-ring benzoporphyrin showed no tumor control, while the A-ring derivative showed complete tumor regression at 320 days. It must be noted that upon injection the solution was diluted to such an extend that the polymer concentration decreased below its $\mathrm{CMC}$, and the photosensitizer was, therefore, expected to be released from the micelles and be taken up by tumor cells through binding with plasma proteins. The reduced efficacy of the B-ring benzoporphyrin may be explained by the tendency to self-associate when released from the micelles [27]. Unfortunately, no compari- son was made with the PDT efficiency of, e.g. DMSO solutions or Cremophor EL formulations of benzoporphyrin.

\section{3. pH-responsive micelles}

As mentioned in the Introduction, it would be advantageous in several treatments including photodynamic therapy if a drug delivery system is used that responds to a stimulus in order to release the drug (photosensitizer) selectively at the target site. Introducing $\mathrm{pH}$-sensitivity would be a valuable approach, since it is known that for example tumors and inflamed tissues exhibit a decreased extracellular pH [28]. Moreover, after cellular uptake, the carrier may end up in cellular compartments such as endosomes/lysosomes that exhibit an acidic $\mathrm{pH}$. As a consequence, the polymer polarity and structure may change causing destabilization of the endosomal membranes and/or release of the photosensitizer [29,30].

Following the above approach, $\mathrm{pH}$ triggered photosensitizer release is a method that has been suggested by Leroux et al. [31-33] and more recently by the group of Kataoka [34,35] using polymeric micelle formulations. The following sections summarize their results.

\subsection{Polymers based on poly(N-isopropylacrylamide)}

Leroux et al. used a poorly water soluble aluminum chloride phthalocyanine (AlCIPc) as the photosensitizer and applied random copolymers as the carrier composed of $N$-isopropylacrylamide (NIPA), methacrylic acid (MAA, typically $3-5 \mathrm{~mol} \%$ ) to create $\mathrm{pH}-$ sensitivity, octadecyl acrylate (ODA, $2-4 \mathrm{~mol} \%$ ) to induce micelle formation, and $\mathrm{N}$-vinyl-2-pyrolidone (VP, $8 \mathrm{~mol} \%$ ) to enhance hydrophilicity of the copolymer. NIPA and VP copolymers are suitable as the hydrophilic segments in polymeric micelles as they have been reported to reduce absorption of plasma proteins $[36,37]$.

\subsubsection{Micelle formation and drug loading}

The above mentioned NIPA/MAA/ODA copolymers with or without VP had a CMC of about $10 \mathrm{mg}$ $1^{-1}$ (a factor of two lower compared with pluronic $\mathrm{P} 123)$ in water and PBS buffer [31,32]. Micelles 
obtained by dialysis of organic polymer solutions against aqueous phases had sizes in the range of 13$35 \mathrm{~nm}$ at a concentration of $5 \mathrm{~g}^{-1}$ at $20^{\circ} \mathrm{C}$. At these high concentrations, the micelle size was quite sensitive to the environment at the more relevant temperature of $37{ }^{\circ} \mathrm{C}$; in water the particle size remained 19 $\mathrm{nm}$, while in PBS severe aggregation occurred probably due to a salting out effect. At $0.5 \mathrm{~g}^{-1}$ the aggregation was less pronounced [31].

AlClPc loading was carried out by a dialysis procedure against water from DMF solution, in the presence of a copolymer containing $2 \mathrm{~mol} \%$ ODA. Drug loadings of about 3\% (w/w) were achieved, corresponding with a more than 1000-fold increase in AlClPc solubility in water $[31,32]$. The phthalocyanine appeared to be present in its aggregated form in the micelles, which is not preferred in view of the low quantum yields of light absorption of aggregated photosensitizers.

Similar copolymers as those described above, but with two octadecyl chains attached at one terminus of the polymer chain instead of ODA randomly distributed along the polymer chain, showed higher CMC's (20-33 $\left.\mathrm{mg}^{-1}\right)$ and lower drug loading efficiency, indicating the importance of using the proper polymer structure $[32,33]$.

\subsubsection{In vitro evaluation}

The $\mathrm{pH}$-sensitive copolymers were found to be less toxic than Cremophor EL in vitro. No dark toxicity was observed in cell cultures (EMT-6 mouse mammary tumor cells) with all AlClPc copolymer formulations at the maximal concentrations tested, i.e. $10 \mu \mathrm{M}$ AlClPc and $0.22 \mathrm{mg} \mathrm{ml}^{-1}$ polymer [31,33]. Upon light treatment the micelle formulations induced greater photoactivity than a Cremophor EL-based formulation (Fig. 3), probably because of a higher cellular uptake and/or more efficient intracellular localization. Again, the terminally alkylated copolymer appeared to be significantly less efficient than its random counterpart.

It was shown that the presence of $5 \mathrm{~mol} \%$ MAA in the copolymers caused the polymers to precipitate and the hydrophobic core to distort as the $\mathrm{pH}$ decreased below $5.7-5.8$ at $37{ }^{\circ} \mathrm{C}$ [31]. This phenomenon could cause release of the entrapped photosensitizer and change the intracellular localization of the drug in a favorable way. To determine whether the endosomal decrease in $\mathrm{pH}$ plays a role in the observed enhanced

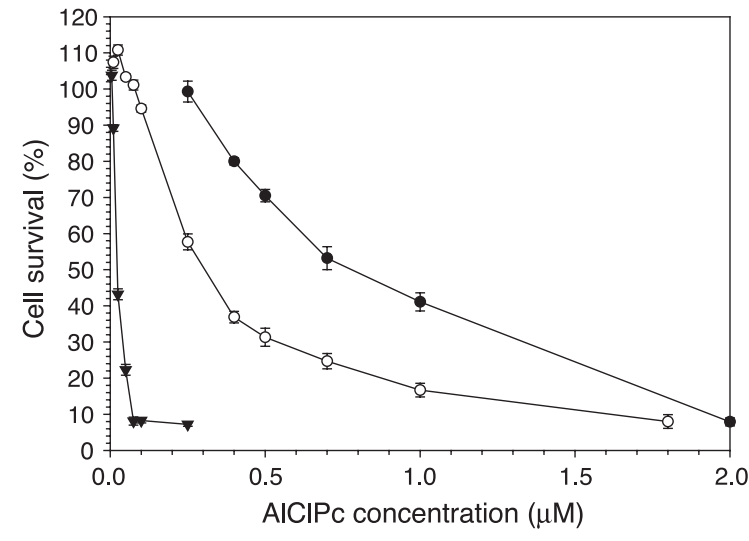

Fig. 3. Survival of EMT-6 cells upon PDT treatment after $1 \mathrm{~h}$ of incubation with AlClPc loaded micelles. Randomly alkylated copolymer $(\boldsymbol{\nabla})$; terminally alkylated copolymer (O); Cremophor EL (๑). (Reproduced with permission from [33].)

photoactivity, cell survival experiments were carried out in the presence of chloroquine, a weak base that is known to raise the internal $\mathrm{pH}$ of acidic organelles [33]. Indeed, in the presence of chloroquine, the activity of the drug loaded in the $\mathrm{pH}$-sensitive micelles decreased, whereas it remained unchanged in the case of the Cremophor EL formulation (Fig. 4).

\subsubsection{In vivo evaluation}

The pharmacokinetics and biodistribution of AlClPc after i.v. administration were determined using EMT-6 tumor-bearing mice [32,33]. Unexpectedly, the polymeric micelle formulations performed worse when compared with Cremophor EL formulations. A more rapid blood clearance, higher liver and spleen uptake, and lower AlClPc levels in the tumor was observed using any of the polymeric micelles. The most hydrophobic copolymer gave high drug levels in the lung after $3 \mathrm{~h}$, which was explained by aggregation of the micelles and, therefore, embolization in lung capillaries. The more hydrophilic copolymers, i.e. those containing VP, showed less spleen and liver uptake than in the absence of VP comonomer. However, the VP containing polymers also showed a higher lung accumulation but only after $24 \mathrm{~h}$. Therefore, lung embolization was ruled out, but another explanation was not given so far. Levels of tumor uptake were similar for all polymeric micelle formulations.

Despite the rather disappointing biodistribution data, animals that were photodynamically treated 24 
h post i.v. administration of polymeric micelle formulations (AlClPc dose of $0.25 \mu \mathrm{mol} \mathrm{kg}{ }^{-1}$ ) showed complete tumor regression in $\geq 85 \%$ of the animals, being comparable with Cremophor EL formulations [33]. The presence of VP as a comonomer in the polymers seemed to have a slightly improved therapeutic effect [32].

\subsection{Polyion complex micelles}

The group of Katoaka reported that upon mixing aqueous solutions of a polycationic porphyrin dendrimer shown in Fig. 2 and a polyanionic PEGpoly(aspartic acid) block copolymer (PEG- $b$-PAA), highly stable micelles are formed based on electrostatic and hydrogen bonding interactions [34], so called PIC micelles [6]. The dendritic porphyrins (DPs) were present as non-aggregated species inside the micelles [35]. The hydrodynamic diameter of the narrowly dispersed spherical micelles was $56.0 \mathrm{~nm}$ at physiological conditions as determined by dynamic light scattering. However, upon increasing the $\mathrm{pH}$ above 7.4 or decreasing below 6.2 the average diameters of the micelles increased to approximately $90 \mathrm{~nm}$ with increased polydispersity. This is caused by decreasing electrostatic interactions due to protonation of the PAA block at low $\mathrm{pH}$ and deprotonation of the dendrimer at high $\mathrm{pH}$, respectively, resulting in a change in the compact core-shell structure. This phenomenon may

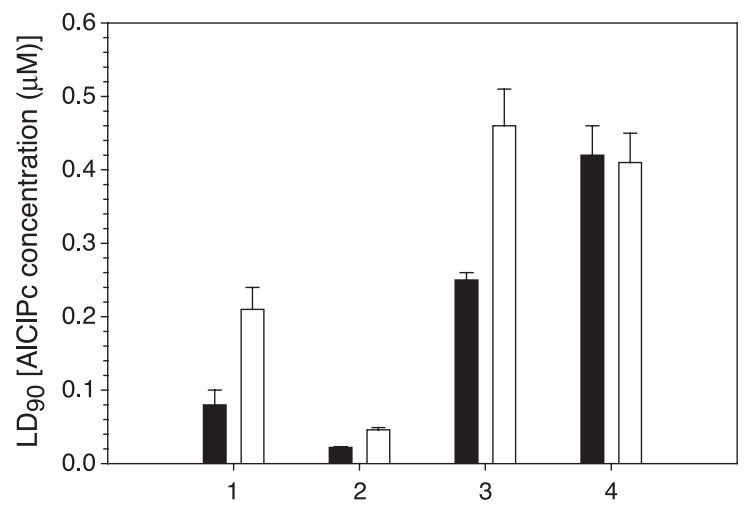

Fig. 4. Dose of $\mathrm{AlClPc}$ in micelles required to inactivate $90 \%$ of EMT-6 cells $\left(\mathrm{LD}_{90}\right)$ upon PDT treatment after $24 \mathrm{~h}$ of incubation with (white bars) or without (black bars) $50 \mu \mathrm{M}$ chloroquine. Randomly alkylated ( $2 \mathrm{~mol} \%$ ) copolymer containing $5 \mathrm{~mol} \%$ (1) and 3 mol\% MAA (2); terminally alkylated copolymer (3); Cremophor EL (4). (Reproduced with permission from [33].)
Table 1

The photodynamic effect $\left(\mathrm{IC}_{50}\right.$, unit: $\left.\mu \mathrm{M}\right)$ of PIX, PIC micelle, and DP in the LLC cell line (adapted from [35])

\begin{tabular}{llll}
\hline & PIX & DP & Micelle \\
\hline $4 \mathrm{~h}$ & 4.26 & 0.403 & 0.0289 \\
$12 \mathrm{~h}$ & 1.67 & 0.327 & 0.0275 \\
\hline
\end{tabular}

be exploited for intratumoral or intracellular endosomal delivery of the photosensitizer as explained above.

In vitro studies revealed that the PIC micelles were internalized in Lewis lung carcinoma (LLC) cells by the endosomal pathway [35]. Basically no dark-toxicity was observed. As shown in Table 1 the micellar formulation exhibited the highest photodynamic efficacy in terms of $\mathrm{LD}_{50}$ values on LLC cell viability when compared to the porphyrin dendrimer alone or protoporphyrin IX (PIX), despite the observed lower uptake of the micelles. This may be ascribed to the lower tendency of the porphyrin to aggregate in the micelles and/or its improved intracellular localization. No in vivo results have been reported yet.

\section{Summary and future prospects}

The results presented by Leroux et al. reveal the high potency of AlClPc polymeric micelles when localized in tumor tissue, which can probably be attributed to the $\mathrm{pH}$-sensitivity of the polymers causing an improved intracellular distribution of the photosensitizer. Therefore, polymeric micelle formulations are good alternatives for Cremophor EL, since the latter is a relatively toxic additive [38]. However, the biodistribution characteristics of the polymeric micelle formulations investigated so far are clearly susceptible to improvements. For example, in contrast to the polymeric micelle formulations presented here, it was shown that PEG-PLA polymeric micelles mediated circulation times of paclitaxel being similar to Cremophor EL paclitaxel formulations and tissue and tumor levels being $2-3$-fold higher at the maximum tolerated doses [9]. Also, Kataoka et al. described long-circulating surface-modified PEG-PLA micelles showing $25 \%$ of injected dose still circulating at $24 \mathrm{~h}$ after administration [39], whereas the NIPA-based micelles described above were completely cleared at that time [33]. 
Thus, many opportunities still exist to optimize the otherwise promising $\mathrm{pH}$-responsive polymeric micelle system. One could pay attention on introducing targeting ligands, and site-controlled release capabilities other than $\mathrm{pH}$-induced release. The polymer and photosensitizer could be further optimized using degradable systems, which would be advantageous in terms of the final clearance of the compounds from the body to prevent accumulation and long-term toxic effects.

\section{References}

[1] H. Bader, H. Ringsdorf, B. Schmidt, Water soluble polymers in medicine, Angew. Makromol. Chem. 123/124 (1984) $457-485$.

[2] V.P. Torchilin, Structure and design of polymeric surfactantbased drug delivery systems, J. Control. Release 73 (2001) $137-172$.

[3] K. Kataoka, A. Harada, Y. Nagasaki, Block copolymer micelles for drug delivery: design, characterization and biological significance, Adv. Drug Deliv. Rev. 47 (2001) 113-131.

[4] M.L. Adams, A. Lavasanifar, G.S. Kwon, Amphiphilic block copolymers for drug delivery, J. Pharm. Sci. 92 (2003) $1343-1355$.

[5] M.C. Jones, J.-C. Leroux, Polymeric micelles - a new generation of colloidal drug carriers, Eur. J. Pharm. Biopharm. 48 (1999) 101-111.

[6] Y. Kakizawa, K. Kataoka, Block copolymer micelles for delivery of gene and related compounds, Adv. Drug Deliv. Rev. 54 (2002) 203-222.

[7] S.A. Hagan, A.G.A. Coombes, M.C. Garnett, S.E. Dunn, M.C. Davies, L. Illum, S.S. Davis, S.E. Harding, S. Purkiss, P.R. Gellert, Polylactide-poly(ethylene glycol) copolymers as drug delivery systems: 1 . Characterization of water dispersible micelle-forming systems, Langmuir 12 (1996) 2153-2161.

[8] K. Yasugi, Y. Nagasaki, M. Kato, K. Kataoka, Preparation and characterization of polymer micelles from poly(ethylene glycol) - poly(D,L-lactide) block copolymers as potential drug carrier, J. Control. Release 62 (1999) 89-100.

[9] S.C. Kim, D.W. Kim, Y.H. Shim, J.S. Bang, H.S. Oh, S.W. Kim, M.H. Seo, In vivo evaluation of polymeric micellar paclitaxel formulation: toxicity and efficacy, J. Control. Release 72 (2001) 191-202.

[10] I.G. Shin, S.Y. Kim, Y.M. Lee, C.S. Cho, Y.K. Sung, Methoxy poly(ethylene glycol)/ $/$-caprolactone amphiphilic block copolymeric micelle containing indomethacin: I. Preparation and characterization, J. Control. Release 51 (1998) 1-11.

[11] A. Lavasanifar, J. Samuel, G.S. Kwon, Micelles of poly(ethylene oxide)-block-poly( $N$-alkyl stearate L-aspartamide): synthetic analogues of lipoproteins for drug delivery, J. Biomed. Mater. Res. 52 (2000) 831-835.

[12] T. Nakanishi, S. Fukushima, K. Okamoto, M. Suzuki, Y.
Matsumura, M. Yokoyama, T. Okano, Y. Sakurai, K. Kataoka, Development of the polymer micelle carrier system for doxorubicin, J. Control. Release 74 (2001) 295-302.

[13] Y.I. Jeong, J.B. Cheon, S.H. Kim, J.W. Nah, Y.M. Lee, Y.K. Sung, T. Akaike, C.S. Cho, Clonazepam release from coreshell type nanoparticles in vitro, J. Control. Release 51 (1998) 169-178.

[14] J.E. Chung, M. Yokoyama, M. Yamato, T. Aoyagi, Y. Sakurai, T. Okano, Thermo-responsive drug delivery from polymeric micelles constructed using block copolymers of poly $(N$-isopropylacrylamide) and poly(butylmethacrylate), J. Control. Release 62 (1999) 115-127.

[15] D. Neradovic, C.F. van Nostrum, W.E. Hennink, Thermoresponsive polymeric micelles with controlled instability based on hydrolytically sensitive $N$-isopropylacrylamide copolymers, Macromolecules 34 (2001) 7589-7591.

[16] G. Storm, S.O. Belliot, T. Daemen, D.D. Lasic, Surface modification of nanoparticles to oppose uptake by the mononuclear phagocyte system, Adv. Drug Deliv. Rev. 17 (1995) 31-48.

[17] H. Maeda, J. Wu, T. Sawa, Y. Matsumura, K. Hori, Tumor vascular permeability and the EPR effect in macromolecular therapeutics: a review, J. Control. Release 65 (2000) 271-284.

[18] G. Kwon, S. Suwa, M. Yokoyama, T. Okano, Y. Sakurai, K. Kataoka, Enhanced tumor accumulation and prolonged circulation times of micelle-forming poly(ethylene oxide-aspartate) block copolymer-adriamycin conjugates, J. Control. Release 29 (1994) 17-23.

[19] S. Vinogradov, E. Batrakova, S. Li, A. Kabanov, Polyion complex micelles with protein-modified corona for receptormediated delivery of oligonucleotides into cells, Bioconjug. Chem. 10 (1999) 851-860.

[20] K. Yasugi, T. Nakamura, Y. Nagasaki, M. Kato, K. Kataoka, Sugar-installed polymer micelles: synthesis and micellization of poly(ethylene glycol)-poly(D,L-lactide) block copolymers having sugar groups at PEG chain end, Macromolecules 32 (1999) 8024-8032.

[21] N. Kumar, M.N.V. Ravikumar, A.J. Domb, Biodegradable block copolymers, Adv. Drug Deliv. Rev. 53 (2001) 23-44.

[22] Y. Katayama, T. Sonoda, M. Maeda, A polymer micelle responding to the protein kinase A signal, Macromolecules 34 (2001) 8569-8573.

[23] Y. Kakizawa, A. Harada, K. Kataoka, Environment-sensitive stabilization of core-shell structured polyion complex micelle by reversible cross-linking of the core through disulfide bond, J. Am. Chem. Soc. 121 (1999) 11247-11248.

[24] M.B. Vrouenraets, G.W.M. Visser, G.B. Snow, G.A.M.S. van Dongen, Basic principles, applications in oncology and improved selectivity of photodynamic therapy, Anticancer Res. 23 (2003) 505-522.

[25] A.V. Kabanov, E.V. Batrakova, V.Y. Alakhov, Pluronic block copolymers as novel polymer therapeutics for drug and gene delivery, J. Control. Release 82 (2002) 189-212.

[26] N. Hioka, R.K. Chowdhary, N. Chansarkar, D. Delmarre, E. Sternberg, D. Dolphin, Studies of a benzoporphyrin derivative with Pluronics, Can. J. Chem. 80 (2002) 1321-1326.

[27] J.X. Zhang, C.B. Hansen, T.M. Allen, A. Boey, R. Boch, J. Control. Release 86 (2003) 323-338. 
[28] M. Stubbs, P.M. McSheehy, J.R. Griffiths, C.L. Bashford, Causes and consequences of tumor acidity and implications for treatment, Mol. Med. Today 6 (2000) 15-19.

[29] O. Meyer, D. Papahadjopoulos, J.-C. Leroux, Copolymers of $\mathrm{N}$-isopropylacrylamide can trigger $\mathrm{pH}$ sensitivity to stable liposomes, FEBS Lett. 42 (1998) 61-64.

[30] J.E. Chung, M. Yokoyama, M. Yamato, T. Aoyagi, Y. Sakurai, T. Okano, Thermo-responsive drug delivery from polymeric micelles constructed using block copolymers of poly $(\mathrm{N}$-isopropylacrylamide) and poly(butylmethacrylate), J. Control. Release 62 (1999) 115-127.

[31] J. Taillefer, M.-C. Jones, N. Brasseur, J.E. van Lier, J.-C. Leroux, Preparation and characterization of $\mathrm{pH}$-responsive polymeric micelles for the delivery of photosensitizing anticancer drugs, J. Pharm. Sci. 89 (2000) 52-62.

[32] D. Le Garrec, J. Taillefer, J.E. van Lier, V. Lenaerts, J.-C. Leroux, Optimizing pH-responsive polymeric micelles for drug delivery in a cancer photodynamic therapy model, J. Drug Targeting 10 (2002) 429-437.

[33] J. Taillefer, N. Brasseur, J.E. van Lier, V. Lenaerts, D. Le Garrec, J.-C. Leroux, In-vitro and in-vivo evaluation of $\mathrm{pH}$ responsive polymeric micelles in a photodynamic cancer therapy model, J. Pharm. Pharmacol. 53 (2001) 155-166.

[34] G.-D. Zhang, N. Nishiyama, A. Harada, D.-L. Jiang, T. Aida, K. Kataoka, pH-sensitive assembly of light-harvesting den- drimer zinc porphyrin bearing peripheral groups of primary amine with poly(ethylene glycol)-b-poly(aspartic acid) in aqueous solution, Macromolecules 36 (2003) 1304-1309.

[35] G.-D. Zhang, A. Harada, N. Nishiyama, D.-L. Jiang, T. Aida, K. Kataoka, Polyion complex micelles entrapping cationic dendrimer porphyrin: effective photosensitizer for photodynamic therapy of cancer, J. Control. Release (2003) (in press).

[36] A. Yamazaki, F.M. Winnik, R.M. Cornelius, J.L. Brash, Modification of liposomes with N-substituted polyacrylamides: identification of proteins adsorbed from plasma, Biochim. Biophys. Acta 1421 (1999) 103-115.

[37] K. Ishihara, Y. Iwasaki, S. Ebihara, Y. Shindo, N. Nakabayashi, Photoinduced graft polymerization of 2-methacryloyloxyethyl phosphorylcholine on polyethylene membrane surface for obtaining blood cell adhesion resistance, Coll. Surf. B Biointerf. 18 (2000) 325-335.

[38] N. Brasseur, R. Ouellet, C. La Madeleine, J.E. van Lier, Water-soluble aluminium phthalocyanine-polymer conjugates for PDT: photodynamic activities and pharmacokinetics in tumour bearing mice, Br. J. Cancer 80 (1999) $1533-1541$.

[39] Y. Yamamoto, Y. Nagasaki, Y. Kato, Y. Sugiyama, K. Kataoka, Long-circulating poly(ethylene glycol)-poly(D,L-lactide) block copolymer micelles with modulated surface charge, J. Control. Release 77 (2001) 27-38. 